\title{
AVATARES Y FANTASÍAS NEOREALES EN LA GIUDAD VIRTUAL
}

\section{AVATARS AND NEOREAL FANTASIES IN VIRTUAL GITY}

\section{JAVIER GAYO SANTACECILIA}

Coordinador Académico en U-Tad

Edificio Madrid, Complejo Europa Empresarial,

Calle Playa de Liencres, 2 bis, 28290 Las Rozas, Madrid

TIfn: + 0034900373379

javier.gayo@u-tad.com

\section{ENRIQUE MORALES CORRAL}

Cuerpo Académico en U-Tad

Edificio Madrid, Complejo Europa Empresarial,

Calle Playa de Liencres, 2 bis, 28290 Las Rozas, Madrid

TIfn: + 0034900373379

enrique.morales@u-tad.com

\section{PALABRAS CLAVES}

Ciudad Virtual, Realidad Aumentada (RA), Interactividad, Personalización, Neorealidad, Percepción de la Realidad, Smartcity

\section{KEY WORDS}

Virtual City, Augmented Reality (RA), Interactivity, Customization, Neoreality, Perception of Reality, Smartcity 


\section{Resumen}

La creación y "customización" de los universos personales en los entornos tecnológicos de los usuarios ha sido una constante en las últimas décadas. La incorporación de la Realidad Aumentad (RA) a la vida diaria abre una nueva puerta a la reconstrucción de los espacios y las interacciones en la ciudad desde la fantasía Neoreal (Gayo y Morales, 2016), proyectando las necesidades, los deseos y sueños de las personas sobre el tejido tecnológico y social.

El objetivo de esta comunicación es analizar y proyectar, desde el marco de implantación social y tecnológico, un nuevo modelo teórico de interacción entre la realidad y la virtualidad, al abandonar esta última la limitación espacial y temporal que ha tenido hasta el momento, para incorporarse a la vida diaria desde la ubicuidad cotidiana. La mejora constante en el uso y acceso a servicios e interacciones online de los ciudadanos, ha provocado una demanda creciente de comunicación con las instituciones, tanto públicas como privadas, con el objetivo de mejorarla y facilitarla. Una vez superada la barrera tecnológica de acceso y procesado, el usuario es libre de personalizar su realidad desde la integración aumentada de los universos personales, tanto profesionales como de ocio, normalizando la presencia virtual en los espacios reales.

La generación y combinación constante desde la realidad aumentada de nuevas capas personales de neorealidad, plantea una ruptura en la constante perceptiva común de la realidad, para adaptar la misma a la voluntad individual, grupal o corporativa, generando una experiencia y apreciación personalizada de la vida en la ciudad.

\section{Abstract}

The creation and personalization of personal universes in users technological environments has been a constant in the last decades. The incorporation of Augmented Reality (RA) into everyday life opens a new door to the reconstruction of spaces and interactions in the city from the neoreal fantasy, projecting the needs, desires and dreams on the technological fabric and Social. The objective of this communication is to analyze and project, from the social and technological implementation framework, a new theoretical model of interaction between reality and virtuality, leaving behind the spatial and temporal limitation that virtuality has had so far, to incorporate it to everyday life from the everyday ubiquity. The constant improvement in use and access of citizens to online services and interactions has led to a growing demand for communication with institutions, both public and private, with the aim of improving and facilitating it. Once the technological barrier of access and processing is overcome, the user is free to personalize his reality from the increased integration of personal universes, both professional and leisure, normalizing the virtual presence in the real spaces. The generation and constant combination from the augmented reality of new personal layers of neoreality, raises a break in the constant common perception of reality, to adapt it to the individual, group or corporate will, generating a personalized experience and appreciation of life in the city 


\section{Introducción}

En un contexto social próximo de normalización urbana y cotidiana de la percepción y personalización combinada de elementos virtuales y reales dentro de la vida de la smartcity, debemos marcarnos como objeto de estudio el cambio del paradigma cognitivo en la construcción del imaginario social y personal que dicha convivencia plantea, desde la combinación de experiencias neoreales que conlleva la realidad mixta, para ello es necesario proceder a través del análisis de escenarios con el propósito de proyectar opciones y consecuencias.

\section{Objetivos}

El objetivo fundamental de la presente comunicación se centra en el análisis del marco socio-tecnológico de implantación y personalización de la Realidad Mixta para proceder al planteamiento y la proyección de un nuevo modelo teórico de interacción entre Realidad y Virtualidad, que se incorpora de forma ubicua en la smartcity y en nuestra vida diaria personal.

\section{Metodología}

Mediante un análisis bibliográfico se procederá a establecer el marco presente de implantación e integración tecnológico-social de la virtualidad, fundamentalmente en los espacios tecnológicos urbanos, planteando a su vez una serie de proyecciones sobre las vías de interacción personalizada de la realidad y la virtualidad. El proceso se acompañará con datos secundarios que fundamenten los procesos económicos y de integración social, a través de informes fundamentados de importantes firmas tecnológicas.

\section{Realidad Mixta, Tecnología e Imaginarios Sociales}

La construcción del concepto de la realidad más allá de la apreciación perceptiva y psicológica-identitaria unipersonal, se forja también desde la construcción socio-natural (Sandoval, 2010) así como a través del imaginario social como ya apuntaba Jose Luis Pintos (2005) en sus estudios, donde nos planteaba que los imaginarios sociales se definían como representaciones colectivas que regían la identificación e integración social, pero igualmente nos indicaba era a través de ellos como podíamos percibir algo como real, y llegar a explicarlo al intervenir operativamente en lo que en cada sistema social considera como realidad.

Hasta ahora los universos o imaginarios personales se construían desde la realidad en lo lógicoperceptivo y desde la fantasía o proyección imaginaria en lo semántico-conceptual, en este sentido la aceptación de las proyecciones fantásticas era fundamentalmente narrativa a través de la suspensión voluntaria de la incredulidad, ya que existía una frontera extradiegética más nítida con la realidad. Sin embargo desde el 
momento en que la virtualidad interactiva irrumpió en nuestra sociedad, se empezó a trazar un puente hacia la mutación de los imaginarios sociales, donde elementos, historias y personajes virtuales, nos interpelaban y comenzaban a ser parte de nuestra experiencia vital, y con ello también de nuestra propia realidad, aunque inicialmente desde lo perceptivo-social este puente presentaba claras limitaciones por la tecnología, la implementación y los costes.

En los últimos años se ha vuelto a incidir en el advenimiento de los universos virtuales y aumentados, amparados por una imparable evolución tecnológica en la que, por primera vez en bastante tiempo, si se aprecia un cambio cualitativo y cuantitativo, en las mejoras en la experiencia de usuario aportadas respecto a intentos anteriores, así como en el margen de implementación social y de mercado.

Según el conocido Informe de la Sociedad de la Información en España Edición 2016, financiado por la Fundación Telefónica, el mercado de la RV alcanzará los 15.000 millones de euros para el 2020 (Fundación Telefónica, 2016). Este volumen será generado por experiencias cada vez más inmersivas de videojuegos, películas y experiencias en directo, como conciertos o espectáculos, mientras que la RA, debido a sus grandes posibilidades de integración en el mundo real alcanzará, y según el mismo informe, la asombrosa cifra de 120.000 millones de dólares para el 2020 (Fundación Telefónica, 2016), al tener la capacidad de introducirse mucho más fácilmente en nuestras rutinas diarias y adaptarse en negocios ya estructurados y consolidados, como el comercio electrónico, elementos publicitarios de visionado e interacción, comunicación personal, elementos culturale

Así podemos ver un interés y apoyo comercial claro por parte de numerosas empresas hacia la experiencia virtual, encontrando incluso la búsqueda de cierta estandarización desde los sistemas o los contenidos, pero a la vez con mejoras de los dispositivos de inmersión e interacción. Éste es el caso de Samsung con las Gear VR ${ }^{1}$, que ahora además de incorporar un mando de control, como ya tenían las HTC $V_{i v e}{ }^{2}$, se plantea que su contenido será compatible con las Oculus $\mathrm{Go}^{3}$; cuya búsqueda de liberación respecto al móvil u ordenador, coincide a su vez con la HTC Vive, que incluso analiza con sus cámaras el espacio para controlar mejor las experiencias. Como podemos observar las conexiones se multiplican y las posiciones de las empresas se acercan hacia ciertos estándares, a la vez que tratan de superarse entre ellas.

Desde el software, son muchas las compañías que además apuestan fuerte no solo por la Realidad Virtual, sino Aumentada e incluso Mixta, fundamentalmente a través de la compatibilidad o integración con Windows10, como ya era el caso de Oculus Rift ${ }^{4}$, pero tal y como se pudo ver en el IFA 2017 en Berlin ${ }^{5}$, a éstos se suman soluciones de compañías como Lenovo, Asus, Dell, Acer y HP, consiguiendo así una cierta estandarización inicial, sobre la que constantemente cada compañía intenta superponer su propia firma personal y otros avances diferenciadores.

Microsoft como propietario de Windows, marcó un horizonte claro con a través de sus HoloLens ${ }^{6}$, y ahora pretende ampliarlo licenciando la tecnología Windows Mixed Reality ${ }^{7}$ a estos cinco fabricantes principales, generando así una competencia que probablemente haga más asequibles algunos modelos estándar, de cara 
a imponerse cada uno lo más posible en el mercado, lo que sin duda favorecerá claramente su integración social como producto.

Por su parte Google busca la compatibilidad a través del ya más que consolidado mercado móvil mediante Android $\mathrm{VR}^{8}$, y la suma de su Project Tango ${ }^{9}$ para que el sistema reconozca el entorno y objetos cercanos, de forma que se optimice y personalice la experiencia en realidad aumentada y mixta; mientras tanto otras firmas se intentan liberar de la necesidad de dispositivos móviles, apostando por sistemas propios.

Sea como sea el estándar final está claro que todos los fabricantes de tecnología han oído el disparo de salida virtual y anhelan posicionarse en una parte de este entorno, ya que además de estas cinco empresas principales encontramos muchas otras apuestas con modelos propios como es el caso de Zeiss VR One, Avegant Glyph, Razer OSVR, Woxter neo VR1 o las humildes Google Cardboard entre otras.

Así, ya sean elementos tecnológicos creados específicamente para una marca de dispositivo móvil o buscando la compatibilidad de diferentes móviles, el movimiento industrial que generan reafirma el interés general en que esta vez la tecnología virtual, aumentada y mixta se implemente a nivel de consumo masivo y social, aprovechando y potenciando el entramado tecnológico que proveen los servicios de la Smartcity.

La maniobra comercial de NVIDIA con su autoconcedida distinción VR READY ${ }^{10}$, se ha visto respondida con la Oculus Ready de Asus ${ }^{11}$, o con la AMD Radeon VR Ready ${ }^{12}$. Indicando que ningún fabricante quiere quedarse fuera de la explotación e implementación del VR.

Evidentemente el universo de los videojuegos forma parte inseparable de este desembarco, desde la aparición de Sony Playstation $\mathrm{VR}^{13}$ y su reciente actualización en mercado, que plantea renovar la experiencia del jugador, hasta la iniciativa de la plataforma de juego Steam, que por su parte planteó también con Steam $V^{14}{ }^{14}$ la experiencia fuera de la pantalla y a escala de "cuarto de juegos", lo que permite al jugador moverse y disfrutar las experiencias lúdicas desde una perspectiva diferente.

En este sentido es muy destacable que se empieza también a tener en cuenta el espacio real desde la virtualidad, de manera que si el usuario se acerca demasiado a un límite en el mundo real estando inmerso en el virtual, su sistema le avisará mediante una interacción en el mundo virtual, algo en consonancia con la dirección tomada por Windows Mixed Reality.

Pero el planteamiento que nos interesa va más allá de las experiencias lúdicas guiadas o limitadas, sino que se combina y autoalimenta con la fusión de la vida real-virtual de los usuarios. En esta dirección Samsung a través de su Odyssey VR ${ }^{15}$, en la línea marcada por Microsoft, busca que los usuarios puedan personalizar su hogar/oficina virtual con contenido seleccionado por ellos o de propia creación, abarcando desde el trabajo al ocio y las preferencias personales, tanto en lo referente a cultura, decoración, deportes o viajes entre otros campos. 
Mientras tanto, afianzando el peso de su extensa red social y sus usuarios, Facebook por ejemplo, ha apostado por la socialización virtual desde FaceBook Spaces ${ }^{16}$ donde mediante avatares creados desde la caricaturización automática de imágenes reales de sus usuarios, plantea nuevas vías de interacción virtual, lo que para algunos sería sorprendente, pero para otros solo es una continuación natural de su vida, como parece ser el caso de la llamada "Generación Z", que perfila y analiza Cerezo (2016) comentando cómo se definen por adoptar de forma más rápida y natural los avances tecnológicos, tendiendo asimismo a entender la relación personal y social de forma mucho más digital y virtual.

Lo cierto es que más allá del perfeccionamiento tecnológico en la inmersión perceptiva o en la búsqueda, o no, de la mímesis en la imitación virtual-real, el otro avance claro respecto a los intentos de implantación de la virtualidad de las décadas de los 90 del XX y la primera década del XXI, es la apreciación y aceptación social de la combinación de la realidad Virtual y Aumentada en la Realidad Mixta, como evolución natural en la constancia social y perceptiva desde la integración tecnológica.

Es en este contexto en el que la realidad personal pasa a transformarse de forma líquida y constante a través de capas superpuestas o coexistentes de realidad mixta que se complementan conformando el imaginario personal y la interacción social, en lo que venimos llamando Neorealidad o Espacio NeoReal. (Gayo, Morales 2016). Es lo que Castells (2006) siempre ha defendido, la conjunción del yo real y el virtual, pero llevado a límites tecnológicos solo soñados donde las percepciones se confunden.

Vemos así anuncios como la campaña de publicidad de noviembre de 2017 de Caser Seguros, realizada por Saatchi \& Saatchi ${ }^{17}$, en el que aún en clave de humor se empiezan a mencionar como comunes, experiencias y problemáticas de integración de la virtualidad en nuestras vidas, dejando claro que la realidad virtual y mixta forman parte ya del imaginario social actual, invitando a reflexionar o plantear desde distintos ángulos, las consecuencias de su implementación masiva, en este caso desde algo tan inicialmente alejado de la virtualidad en nuestras mentes como un seguro de hogar.

Pero volviendo al concepto de realidad, lo cierto es que independientemente de la ventana o marco tecnológico a través del que nos asomamos o entramos ahora a estos universos virtuales, desde mucho antes podíamos plantear que la realidad es un constructo, en el sentido que aceptamos como "reales" elementos y personajes dentro de un contexto. Así Aguilar (2010) nos indicaba que la propia realidad virtual no es en realidad artificial, sino que desde la intervención mediática conforma una retopología del entorno para ser percibida como real.

Bajo ese enfoque la integración de elementos virtuales dentro de la realidad de los que se vale la Realidad Aumentada, se replantean desde la interacción y la experiencia de usuario, como componentes válidos en un contexto y por tanto "reales" en cuanto a su sentido y utilidad en nuestra relación con ellos.

De igual manera observándolo desde su efecto en las reacciones y los sentimientos que despierta, el impacto emocional de determinadas interacciones virtuales o mixtas es indistinguible del que provocan 
interacciones con elementos o situaciones reales, incluso sin depender de la mímesis hiperrealista, confirmando la afirmación de Echevarría de la imposibilidad de equiparar lo "artificial" con "irreal". (Echeverría, 2000)

Aun así, la Realidad Virtual funciona de forma similar a como lo hacía la narración audiovisual, desde la "suspensión de la incredulidad" conduciéndonos hacia una aceptación emocional que nos absorbía en la pantalla, pero en este caso amplificada a través de la inmersión sensorial y la interacción que ofrece la virtualidad, sin embargo estas ensoñaciones o duermevelas, permanecían encerradas tras el aislamiento del mundo real que proveía la venda en los ojos que proporcionaba el casco de inmersión.

Con la aparición de la Realidad Aumentada empezamos a extraer del escenario, de la pantalla aislada, a los actores y elementos virtuales para recolocarlos a nuestro lado en el mundo real, es entonces cuando la ensoñación cobra vida en el mundo real, y por tanto está formando parte de él y de nuestra realidad desde lo semántico y lo interactivo.

A través de la liberación tecnológica de los límites espaciales iniciales que imponía la Realidad Virtual, la suma de ésta con la Realidad Aumentada conforma la Realidad Mixta, donde determinados elementos reales y virtuales se combinan desde la consolidación y aceptación en el imaginario social, pudiendo reconstruirse de forma constante en la apreciación personal y social, pero a su vez potenciado a través de la red tecnológica urbana pudiéndonos interpelar desde cualquier ubicación.

\section{Ubicuidad, integración y espacialización}

Siempre es arriesgado realizar una Prognosis del futuro, sobre todo cuando los imparables avances en todos los campos, hacen que las líneas de nuestra civilización puedan implosionar, o escindirse en direcciones ni siquiera imaginadas. Pero si echamos un vistazo atrás, veremos que aunque las tecnologías cambien, en todas ellas hay una constante, nosotros, el ser humano.

Precisamente analizando cómo nos hemos ido adaptando, comportando y definiendo en las demás progresiones tecnológicas, y a la vez observando cómo funciona nuestro tejido de interrelación social desde la tecnología, podemos quizá atisbar líneas que van definiendo nuestra relación con las mismas. En este sentido Costa (2014) nos plantea que las singularidades del medio móvil se forjan desde la integración multimedia, la personalización, la geolocalización y la participación. Revisemos la integración y combinación de estos conceptos.

La revolución móvil permitió inicialmente aumentar nuestras capacidades de comunicación, liberándonos del hilo conector para tener la libertad de contactar con cualquiera en cualquier momento, conectividad social que se multiplicó exponencialmente con los sistemas de mensajería y posteriormente videollamada.

Lograda la comunicación la siguiente meta era el acceso a contenidos y el entretenimiento, es decir la integración multimedia. Fusionando internet con nuestros dispositivos móviles los juegos, música y series 
online pasaron de estar a un clic de ratón, para estar a una pulsación táctil de distancia, permitiendo al usuario-consumidor acceder a sus elementos de ocio favorito en cualquier momento y sitio incluidos los juegos multijugador y por supuesto las redes sociales.

Es en este instante de capacidad conectiva donde entran en juego la geolocalización y la participación. Mediante la identificación del lugar donde está el usuario, se permitió a numerosas apps multiplicar sus servicios potenciando así en el usuario las interrelaciones o posibilidades a su alcance, tanto a nivel comercial como personal y potenciado el tejido interactivo social.

Pero es desde el momento en que se empieza a escanear el espacio donde está usuario cuando la interacción alcanza otras cotas. Lo que de manera simple empezaron haciendo las tecnologías Kinect (Microsoft), WiiMote (Nintendo) o Psmove (Sony), como reconocimiento del propio usuario, ha evolucionado cuando las tecnologías de realidad mixta son ya capaces de empezar a explorar y documentar interactivamente el espacio real donde se encuentra el usuario.

Aquí se multiplican las ensoñaciones de marketing que ya buscaban la microetiquetación del consumidor desde el Big Data y la personalización del consumo informativo y publicitario, dado que el espacio de interacción mixto permite hacer de cada espacio dentro de la smartcity, de cada mirada del usuario/ espectador/consumidor, un detonador virtual e interactivo de intereses comerciales.

Aunque pueda parecer un escenario aún lejano, las bases tecnológicas y sociales ya están cimentadas, en este sentido la adopción e implantación tecnológica es simplemente una cuestión de mercado y normalización, pues como nos decían Alonso y Arzoz (2003), la tecnología forja nuestra sociedad desde tres sentidos: "como realidad, como proyecto y como sueño.", haciendo que de forma retroalimentada encontremos nuestro sentido en ella.

La inmersión prácticamente constante en la realidad mixta, nos plantea no una simple superposición de elementos virtuales o aumentados, sino una multiplicación de nuestras visiones y proyecciones, desde el momento en que ofrecemos y recibimos diferentes visones digitales de nosotros mismos, dependiendo de la ocasión y el interlocutor. Esta visión y proyección a su vez retroalimenta nuestra propia "Weltanschauung", nuestra visión de la realidad, como nos recordaba Sanz (1996) desde el momento en que empleamos un código que se impone al espectador para interpretar una realidad visual.

"La organización de la realidad visualmente percibida, así como la estructuración del pensamiento visual a través del código icónico, son los procesos básicos que condicionan el universo individual en el nivel de la experiencia. La imagen determina el mundo personal, en este aspecto, cuando menos tanto como la palabra." (Sanz, 1996 - Pag 58).

Hoy nos parece habitual y normal que millones de personas cada día intercambien dinero con un gesto en su dispositivo móvil en vez de abrir sus carteras, que hayan acudido en masa en la búsqueda y caza del Pokemon escondido en su entorno, que experimenten visualizando cómo queda su piso real con decoración 
virtual (que posiblemente será real más tarde) o incluso expliquen a sus hijos cómo se comportaba un animal prehistórico viéndolo pasear por su salón.

En este sentido, el camino que se nos imponía hace décadas, desde las visiones futuristas del ciberpunk que nos condenaba al aislamiento sensorial externo en la búsqueda del paraíso virtual, pasa ahora por aceptar como algo natural extraer esos elementos hacia nuestro mundo acompañándonos en nuestro entorno y por nuestras ciudades.

Este desembarco de la virtualidad en nuestros espacios reales que estaba inicialmente constreñido a un área de acción muy limitada puede a través del soporte conectivo móvil expandirse muy rápidamente. Debemos plantearnos ahora desde la implantación y normalización de la Realidad Mixta, qué puede ocurrir entonces cuando la virtualidad empieza a tener la oportunidad, tecnológica y social de acompañarnos en el día a día y de forma ubicua, es decir, no sólo en nuestros hogares sino en nuestras ciudades, al igual que lo hicieron los teléfonos móviles, y con probablemente un potencial de impacto mucho mayor que el cambio relacional y social que los dispositivos móviles nos proporcionaron.

Esto es algo por lo que precisamente apostaban hace décadas autores como Ascott (1998), que en su arquitectura de la ciberpercepción, apoyaba la idea no ya por una sustitución de la ciudad real por la ciberespacial, sino por una interacción o interfaz entre ambas, anticipando precisamente lo que de forma probable sea nuestra apreciación futura de la vida cotidiana de realidad mixta en nuestras ciudades. Al mismo tiempo este nuevo planteamiento espacial, se enfrenta al concepto de la desaparición de los espacios o picnolepsia, que nos advertía Virilio en su Cibermundo (2005), la Realidad Mixta al permitirnos recuperar nuestra propiocepción más allá del aislamiento perceptivo de la realidad virtual, hace que el espacio recupere su sentido, a la vez que lo muta al compartir sus coordenadas con la virtualidad.

De hecho en el momento en que a través de la Realidad Aumentada y Mixta damos cabida a la virtualidad combinada de espacios reales-virtuales, e incluso a la interacción de individuos y avatares, que pueden estar basados en un interactor humano o ser de procedencia totalmente virtual, es cuando podemos considerar que pasamos del concepto de la "Simultaneidad Desespacializada" de Thompson (1998) a lo que podríamos denominar "Espacialización Simultaneada" en la Realidad Mixta.

El tejido tecnológico que se ha ido desplegando por nuestras ciudades para ofrecer inicialmente servicios de comunicación y datos a baja escala, ha crecido en potencia y capacidad, dando un marco de interacción mucho más amplio en lo social o lo personal. Este marco además interactúa desde la ubicuidad en las smartcities, lo cual establece nuevas conexiones entre lo público y lo privado, como apuntaban Domingues y Lucena (2016) "La interconexión ubicua no significa el fin del territorio controlable, o la eliminación de distinciones entre público y privado, sino que nos compele a repensar y reinventar esas construcciones esenciales en un nuevo contexto". Lo cierto es que dicho tejido parece estar ya casi preparado para ofrecernos en los próximos años una experiencia vital inmersiva-interactiva desde la Realidad Mixta, o quizá muchas distintas entre las que cambiar según queramos o necesitemos. 
Esto nos lleva a dos caminos, uno, el impuesto desde la oferta de productos y servicios tanto privados como públicos que la necesidad no negociable de tecnificación plantea. Desde este punto de vista y de forma análoga, muy pocas personas en nuestra sociedad pueden o quieren ya negarse a utilizar en su vida profesional y personal un móvil o internet, salvando cuestiones de acceso económico, lo que nos lleva a una aceptación contractual tecnológica constante en el que debemos firmar y actualizar queramos o no, pero donde se nos deja en general aún escoger algunas de sus condiciones.

El otro camino inseparable del anterior, es que esa aceptación no negociable, se viste bajo la premisa de que en realidad no pasamos a formar parte de esa tecnología, sino que esa tecnología es la que pasa a formar parte de nuestro universo propio, desde la personalización y adaptación a nuestros gustos e intereses, por lo que el volcado de nuestra vida en ese entorno se transforma de obligación, en herramienta y ocio, bajo una decisión controlada, o incluso puede transformarse en ansia y vorágine, desde la velocidad social, pero articulándose para ponernos siempre en el centro del escenario, permitiéndonos la elección de nuestro papel, nuestro vestuario y nuestro guión, a través de la personalización. Esto es posible desde el paso de la Customización Masiva hacia la Personalización Absoluta.

\section{Del consumo masivo a la customizacion absoluta}

Durante las últimas décadas nuestra sociedad de consumo ha sufrido un proceso de "Globalización Customizada". Así, como recogía Arbaiza (2013), ya en los años noventa distintos autores centrados en los procesos de consumo, como Pine, Davis, Peppers y Rogers, incidieron en la tendencia al alza de la integración más directa de las opiniones y gustos de los consumidores, desde un nuevo enfoque hacia la individualidad, de forma que abandonada la producción solo para masas, progresivamente se fueron poniendo a disposición del usuario/consumidor, opciones y herramientas para adaptar productos y servicios a sus propias preferencias.

Lo que inicialmente se limitaba a escoger un color de zapatillas o ingredientes de comida rápida, ha evolucionado, gracias fundamentalmente a la sociedad digital, para ir permitiendo al consumidor mapear su mundo personal sobre aquellos productos y herramientas que utiliza.

“(...) se puede apreciar como se han puesto en marcha procesos estructurales en el que todos los aspectos de la organización -personas, procesos, estructuras y tecnología-están orientados a ofrecer a muchos clientes lo que quieren y necesitan prácticamente de manera particular." (Arbaiza. 2013. Pag 189).

En este sentido, sin duda la ubicuidad de acceso tecnológico mediante las comunicaciones móviles, ha decantado y catalizado una reorientación a la customización y el individualismo ya apuntado por el padre del Marketing Directo Lester Wunderman (2010) ${ }^{18}$, quién plantea una sociedad inmersa en un proceso de empoderamiento del consumidor/usuario desde lo que llamó "personal advertising". 
Así pues, tanto la tecnología como el enfoque del uso de la misma, está llevando a la sociedad hacia un orientación perceptiva cada vez más centrada en la propia existencia, tanto desde la vida social construida y compartida en las redes, como desde la proyección retroalimentada de los deseos y opiniones personales que nos piden poder diferenciarnos de la masa y reconstruirnos como concepto social constantemente. Esa personalización y diferenciación nos la planteaba Martucelli.

"Hoy en día, (...) es imperioso reconocer la singularización creciente de las situaciones. Es cada vez menos probable encontrarnos con 'clones' sociológicos, o sea, con individuos que han tenido las mismas experiencias, en los mismos lugares, en los mismos momentos; en breve, con trayectorias gemelas a las nuestras. La diferenciación de experiencias es la regla y tras ella se afirma la expansión de la singularidad" (Martuccelli, 2010. Pag 27).

Así se acaban combinando la auto-reafirmación y la "e-retroalimentación" mediante la sociedad de redes, desde una óptica amparada en un lema vital cercano a "protagonizo y personalizo mis experiencias, luego existo".

Desde el punto de vista del Mercado, el diseño afectivo y la experiencia de usuario se han convertido en valores fundamentales para decantar comportamientos de consumo, al buscar como Indican Feng, Yangjian y Jianxin en las necesidades latentes más allá de las puntuales.

"(...) Al capitalizar la experiencia del usuario, se espera que el diseño afectivo y cognitivo para la personalización en masa satisfaga las necesidades latentes del cliente $(\mathrm{CN})$ del cliente individual". (Feng et al. 2013 Pag. 1047)

En cada una de las oleadas de innovación tecnológica que hemos incorporado a nuestras vidas en las últimas décadas ya habíamos logrado customizar nuestra experiencia, seleccionado nuestros contenidos y aplicaciones, nuestros fondos y tonos, o sencillamente escogiendo la carcasa o funda de móvil/tableta/ dispositivo que nos diferenciara, que personalizara nuestras posesiones, que reflejara nuestros gustos y deseos. Sin embargo uno de los grandes atractivos que nos aportó internet y las redes sociales era precisamente el permitir al usuario reinventarse y redibujarse de forma personal en el reflejo que construía y compartía, pudiendo como proyección de nuestra persona construir un mundo virtual que podía ser realista, parcialmente mimético o totalmente fantástico, pero que ante todo era definitivamente personal. Esta mirada centrada en nosotros mismos y encerrada desde la tecnología, la encontramos en autores como Siegel (2008) o Carr (2014).

“[...] el culto a la popularidad, que ensalza el <<tú>>, nos está infundiendo una impaciencia impulsiva hacia todo aquel o todo aquello que no somos nosotros o es nuestro" (Siegel. Pag 125).

En ese universo digital y virtual nacido entre la cultura participativa y las manifestaciones popularescomerciales, las proyecciones de uno mismo se fusionan generando el multiverso del solipsismo digital, dando lugar en algún momento al iMe, a la fusión de avatares y usuarios absoluta y constantemente en movimiento, 
desde el momento en que atravesada la cuarta pared, al mandar una miríada de representaciones de cada vida a través de las redes, solo queda proyectarlas en el teatro del mundo, derribar las otras paredes y convertir el mundo en escenario. Y precisamente entonces llega la realidad virtual, aumentada y mixta, permitiéndonos trasvasar nuestro universo prosumido a nuestra cotidianeidad en el momento en que la smartcity lo fomenta y utiliza desde lo comercial-social.

Cuando Janet Murray nos hablaba de la holocubierta (1999), las posibilidades tecnológicas apenas habían empezado a bocetar la ubicuidad conectiva. La Virtualidad se planteaba como un concepto anclado a un espacio cerrado y limitado. Los intentos de sumergirnos mediante visores en universos virtuales seguían atados o encadenados por el mismo cable conector y el espacio limitado, pero generaban un hábito, ansia y anhelo en los usuarios, el de dominar la realidad en vez de someterse a ella.

"En estos momentos comienza también a ser realidad que la persona que juega se convierte en creador y diseñador de mundos virtuales, de un poderoso realismo, y personalizables ya en gran medida a gusto del usuario." (Montero, Ruíz y Díaz, 2010, p. 27).

Es la batalla interior de nuestros deseos que nos plantea Odina (2000), entre la pretensión extranatural o irreal del deseo de ser originales frente a nuestro cautiverio biológico. De ahí la tendencia al alza que nos plantea Rogers (2010) acerca de Personalización y la Customización que nos lleva a la integración de la propiocepción social como personaje múltiple. Esto es a su vez fruto de la capacidad/necesidad interpretativa que Gombrich, Hochberg y Black (2007) definen como una combinación entre la imposición y la plasticidad dócil que presenta el hombre en las interacciones sociales, sumada a la acción y deseo de los Prosumidores/ Interactores, que superado el rol de espectadores se apropian ya de mundo narrativos como apunta Scolari (2013).

Acostumbrados a poder adaptar aspectos y contenidos, los usuarios son libres de construir una visión personalizada y participativa, de igual manera que los "Modders" en los videojuegos, como recuerda Baigorri (2010), disfrutan desplazando de su estética o semántica original elementos y espacios, reinventando o reinterpretando contenidos originales, o en este caso reales.

Y es que debemos considerar también que la gamificación se ha incorporado a muy diversos aspectos de nuestra vida profesional y social, este concepto de juego se impone como herramienta útil pero también se llega a plantear en su extremo, como justificación de la presencia del acto lúdico frente a casi cualquier situación o acción, todo es juego, todo es escenario. Esta tendencia unida a la socialización de las redes nos plantea vías para que ese mundo personal a su vez encaje, o se expanda con las visiones o adaptaciones que otros usuarios/creadores afines nos aporten, jugamos con otros, somos espectador y espectáculo. Pues como recogen Hierro, Lopez y castro (2014) es a través de la customización gamificada como los usuarios se expresan y vinculan con otros usuarios, a través de la identificación de elementos de un código común. 
Así la alteridad en las redes ya se plantea desde hace años como algo natural, como nos recuerdan Cáceres, Ruiz y Brändle (2009 pag 227).

"resulta coherente relacionar el entretenimiento y la diversión con "jugar" o "experimentar" con cambios de identidad. Ser otro, ser de otra manera, habla de una estrategia de exploración, descubrimiento, prueba o puesta en escena de múltiples yos que se juegan en las prácticas de ocio y entretenimiento como campo de ensayo de modelos de comportamiento, de experimentación libre de consecuencias o de interacciones inasequibles en la vida real."

En este contexto el jugar a ser otro como la acción diaria en las redes sociales y consumo constante y cambiante de la Sociedad Líquida de Bauman (2007) se multiplica exponencialmente desde la virtualidad y la realidad mixta. Pero ya no sólo nos mutamos nosotros, podemos mutar nuestro entorno. La tecnología de mapeo 3D descrita como Simultaneous Localization and Mapping (SLAM), es capaz de reconocer los objetos que la cámara captura ofreciendo al usuario filtros 3D adecuados a los mismos. De esta forma estos objetos o entornos reales se convierten en elementos del juego o la ficción virtual y los usuarios pueden interactuar con la animación que se superpone a la imagen a tiempo real. La capacidad actual de transformar una fotografía en dos dimensiones en un espacio 3D y tras años supeditados a la pantalla, integrarlo en nuestra percepción del mundo, lo que daría finalmente alas al concepto que Tom Caudell y David Mizell bocetaron como realidad aumentada en los noventa.

$\mathrm{Si}$ bien es cierto que no todos los consumidores/usuarios tienen las mismas tendencias proactivas, la aproximación hacia una tendencia educativa y socio-cultural, tanto desde el ámbito de la formación y la autoformación, donde se intenta capacitar en general de un pensamiento más adaptativo-plástico, por encima de una mera resolución de modelos; está conformando en la sociedad nuevas líneas de pensamiento en busca de la innovación, la exploración lúdica y la creatividad interactiva, validando, o al menos permitiendo así, nuevos puntos de vista y opiniones diversas, frente al determinismo de épocas pasadas, aunque evidentemente para aquellos que lo prefieran, la propia industria proveerá de elementos pre-configurados que nos ofrezcan experiencias satisfactorias.

Si ya se estaba incentivando a través del uso tecnológico, la creación de puntos de vista personales, se está reforzando desde la educación y la interrelación social la consolidación de un multiverso de opinión y percepción, como herramienta de evolución personal y social, independientemente del buen uso que se haga de ello, o la solidez/validez de sus constructos.

\section{Conclusiones}

El ser humano ha adaptado su entorno a sus deseos y necesidades desde el origen de la humanidad, lo que empezó siendo un imperativo de supervivencia o una búsqueda de progresión de la civilización, quedó enmarcado en el torbellino autocomplaciente del consumo, el posmodernismo y prosumismo. 
En lo tecnológico hemos personalizado o customizado todo dispositivo o entorno que utilizamos, desde los primeros iconos y sonidos de escritorio, hasta el aspecto interior y exterior de nuestros dispositivos. Esta customización inicialmente orientada a la propia satisfacción, se multiplica desde el momento en que los propios intereses comerciales, ven en las creaciones de los usuarios una herramienta de marketing, reducción de costes y prolongación de la vida de sus productos, como plantean Gandhi, Magar y Roberts (2014).

"Al permitir que los clientes creen productos reales e virtuales, las empresas pueden, de hecho, utilizar a los clientes como vendedores y co-creadores. Las tecnologías sociales facultan a los clientes para transmitir sus creaciones a una gran red, que es esencialmente marketing gratuito para la compañía cuyos productos están promocionando".

Igualmente hemos personalizado y virtualizado nuestra presencia a través de las redes, y cogido del mundo virtual sólo lo que encajaba con nuestros intereses y anhelos; en una vorágine antropo-egocentrista.

Ahora los cantos de sirena del Big Data hacen que las fronteras y la visión del mundo se plieguen definitivamente a nuestros íntimos deseos y pareceres, ofreciéndonos algorítmicamente lo que ellos saben, incluso mejor que nosotros, que en realidad anhelamos ver, saber, conocer.

En ese escenario del Panóptico Digital Definitivo, millones de personas viven vidas "virtualmente aumentadas" o ficcionadas en las redes a diario, interpretan papeles y roles en un constante baile de máscaras. Ahora tienen la oportunidad de vestirse con las mejores galas del cosplayer digital que han construido y llevar con ellos su mundo virtual a la realidad.

La última transformación no es ya sólo transmitir la imagen que cada uno ofrece al mundo, sino hacer que el mundo mismo, la realidad, se doblegue a la propia visión que tenemos de la existencia o la que hemos construido en la red, en la virtualidad y al mismo tiempo que esta visión nos acompañe en la ubicuidad de la smartcity.

La capacidad técnica es una simple cuestión de mercado y un poco de tiempo. La oportunidad de dar un paso más en la proyección del yo, es simplemente inevitable, posiblemente progresiva al principio, y posteriormente exponencial, como una normalización generacional que pasará por otra brecha digital, la de los usuarios "neoreales" y la de los "simples conectados", como las otras evoluciones sociales y de comportamiento que la propia red nos ha traído.

El hombre digital ha caído, viva el hombre Neoreal. Porqué pasear sin apenas mirar a los demás, con la cabeza inmersa en nuestros dispositivos móviles, con furtivas miradas fuera del display, cuando podemos levantar la mirada y posarla para siempre en la pantalla definitiva, la que nunca tenemos porqué dejar en reposo, la que ve y filtra el mundo como nosotros queremos, y a la vez lo transforma todo. La pantalla Neoreal de la realidad mixta. 


\section{Referencias}

- $\quad$ Aguilar García, Maria Teresa. 2010. Virilio, Tipler y Baudrillard; Ciberespacio y Cuerpo Virtual. Observaciones Filosóficas. ISSN-e 0718-3712, № 10, 2010

- $\quad$ Alonso, Andoni y Arzoz, Iñaki. Carta al homo ciberneticus. Editorial Edaf S.A. Colección Ensayo. Madrid 2003.

- $\quad$ AMD. Radeon VR Ready. Consultado el 5 de noviembre de 2017. Disponible en http://www.amd.com/es/ technologies/vr-ready

- $\quad$ Arbaiza Rodríguez, Francisco (2013). Orígenes de la customización masiva: Las interacciones individuales consumidor marca dentro de mercados masivos en el marketing. Revista de Comunicación 12, 2013. dialnet.unirioja.es

- $\quad$ Ascott, Roy. (1998). La arquitectura de la cibercepción, en: Giannetti, Claudia (ed.). Ars Telematica. Telecomunicación, Internet y Ciberespacio. Barcelona, L'Angelot,

- $\quad$ Asus VR. Consultado el 5 de noviembre de 2017. Disponible en https://www.asus.com/microsite/beyond-vrready/

- $\quad$ Asus Windows Mixed Reality Headset - Explore Your Imagination | ASUS. Consultado el 5 de noviembre de 2017. Disponible en https://www.youtube.com/watch?v=ZwxloWy2wTo

- $\quad$ Baigorri, Laura. (2010). Nuevas interfaces para el arte y el juego. Revista Kepes Año 7 Número 6 EneroDiciembre. 151-165.

- Bauman, Zygmunt:

- 2007, Vida de consumo, Madrid: Fondo de Cultura Económica. ISBN: 9788437506111

- 2007, Tiempos líquidos: vivir en una época de incertidumbre, Barcelona: Tusquets Editores. ISBN: 9788490664025

- $\quad$ Cáceres, M., Ruiz, J. \& Brändle, G. (2009). Comunicación interpersonal y vida cotidiana. La presentación de la identidad de los jóvenes en Internet. CIC Cuadernos e Información y Comunicación, 14, pp. 213-231

- $\quad$ Carr, Nicholas (2014). Atrapados: cómo las máquinas se apoderan de nuestras vidas. Editorial TAURUS

- Caser Seguros 20. Consultado el 5 de noviembre de 2017. Disponible en https://www.youtube.com/watch?time_ continue $=3 \& v=$ OOUWJnR7iW0

- $\quad$ Caser Seguros Campaign by Saatchi \& Saatchi - 11/2017. Consultado el 10 de noviembre de 2017. Disponible en http://www.adsoftheworld.com/campaign/caser-seguros-saatchi-saatchi-11-2017

- $\quad$ Castells, M. (2006) La sociedad red: una visión global. Barcelona: Alianza.

- $\quad$ Cerezo, Pepe. (2016). La Generación Z y la información. En Revista No 114 Los auténticos nativos digitales: ¿estamos preparados para la Generación Z?. Revista de Estudios de Juventud Numero 114. Diciembre 2016.

- Costa-Sánchez, C. Septiembre de 2014. Las singularidades del medio móvil: integración multimedia, personalización, geolocalización y participación. Estudio de su presencia en las apps de la prensa española. Palabra Clave 17 (3), 672-694. DOI: 10.5294/pacla.2014.17.3.5

- $\quad$ Domingues, Diana y Lucena, Tiago. (2016). Reingeniería de la vida urbana [PARTE 1] la extensión de los dispositivos móviles en conexiones ubicuas provoca profundos cambios en la vida urbana, modificando los conceptos de lo público y lo privado en el marco de la cibercultura. Neo Media Lab. Disponible en http://neomedialab.net/ reingenieriadelavidaurbanaparte1/ (Último acceso: 01/12/2017).

- $\quad$ Echevarria Ezpovida, Javier (2000) Un Mundo Virtual. Debolsillo. Plaza y Janés Editores. Barcelona.

- $\quad$ Facebook. Facebook Spaces VR is better with friends. Consultado el 5 de noviembre de 2017. Disponible en https://www.facebook.com/spaces

- $\quad$ Feng Zhou · Yangjian Ji · Roger Jianxin Jiao. J Intell Manuf. Affective and cognitive design for mass personalization: 
status and prospect. (2013) 24:1047-1069 DOI 10.1007/s10845-012-0673-2. Springer Science+Business Media, LLC 2012

- Fundación Telefónica (2016) Informe la sociedad de la información en España. Disponible en: https://goo. gl/5JUVEo (Último acceso: 01/12/2017).

- Gandhi, Anshuk; Magar, Carmen y Roberts, Roger. How technology can drive the next wave of mass customization. Business Technology Office. February 2014. McKinsey\&Company.

- Gayo, Javier. y Morales, Enrique. (2016) De la Holocubierta al Holoverso: Sociedad Virtual y Realidad Aumentada. Comunicación en el Congreso CICIC2016 y recogida en el libro de Actas. Disponible en: https://goo.gl/ithFRz (Último acceso: 01/10/2017).

- Giztab. Las gafas de realidad virtual de Google se renuevan con Android VR. Consultado el 5 de noviembre de 2017. Disponible en http://www.giztab.com/las-gafas-de-realidad-virtual-de-google-se-renuevan-con-android-vr/

- $\quad$ Gombrich, Hochberg y Black, (2007)

- Hierro Mariné, Esther; López Gómez, Silvia y Castro Pena, Maria Luz. Tendencias y estado actual de la gamificación en la producción audiovisual. Recogido en Contenidos digitales en la era de la sociedad conectada. Javier Sierra Sánchez, Daniel Rodrigues Parente. (coordinadores). Biblioteca de Ciencias de la Comunicación. Editorial Fragua. Madrid. 2014

- $\quad$ HTC Vive. Consultado el 5 de noviembre de 2017. Disponible en https://www.vive.com/us/

- IFA. IFA NEXT Innovative Engine. Consultado el 5 de noviembre de 2017.Disponible en http://b2b.ifa-berlin.com/ en/IFA/IFASpecialAreas/IFANEXT/InnovationEngine/

- Martuccelli, Danilo. La individuación como macrosociología de la sociedad singularista. Persona y Sociedad / Universidad Alberto Hurtado. Vol. XXIV / No 3 / 2010 / 9-29.

- Microsoft Hololens. Consultado el 5 de noviembre de 2017. Disponible en https://www.microsoft.com/en-us/ hololens

- $\quad$ Microsoft. Introducing the era of Windows Mixed Reality - In case you missed it Publicado el 4 oct. 2017. Consultado el 5 de noviembre de 2017. Disponible en https://www.youtube.com/watch?v=CjX2HI3pAgo

•

- Montero, Eloisa; Ruiz,Dávila, María; Díaz Tejero, Beatriz. (2010). Aprendiendo con videojuegos. Jugar es pensar dos veces. Narcea, S.A. De ediciones. Madrid.

- $\quad$ Nvidia. Prepárate para la realidad virtual con Geforce® gtx. Consultado el 5 de noviembre de 2017. Disponible en http://www.nvidia.es/object/vr-ready-program-es.html

- Oculus Go. Consultado el 5 de noviembre de 2017. Disponible en https://www.oculus.com/go/

- Oculus Rift. Consultado el 5 de noviembre de 2017. Disponible en https://www.oculus.com/rift/

- Odina, Mercedes. (2000). La Aldea Irreal: La sociedad del futuro y la revolución global. Grupo Santillana de Ediciones, S.A. Aguilar.

- $\quad$ Pintos, Juan Luis. (2005). Comunicación, construcción de la realidad e imaginarios sociales. Revista Utopía y Praxis Latinoamericana / Año 10. № 29 (Abril-Junio, 2005) Pp. 37 - 65. Revista Inter nacional de Filosofía Iberoamericana y Teoría Social / ISSN 1315-5216 CESA - FCES - Universidad del Zulia. Maracaibo-Venezuela.

- $\quad$ Rogers Scott. (2010). Level Up: The guide to great video Game Design. Ed. Wiley.

- Sandoval Moya, Juan. Construccionismo, conocimiento y realidad: una lectura crítica desde la Psicología Social. Revista Mad. $\mathrm{N}^{\circ}$ 23, Septiembre de 2010. Departamento de Antropología. Universidad de Chile. Disponible en: http://www. revistamad.uchile.cl/23/sandoval_04.pdf (Último acceso: 01/12/2017). 
- Samsung Gear VR. Consultado el 5 de noviembre de 2017. Disponible en http://www.samsung.com/global/ galaxy/gear-vr/

- $\quad$ Samsung HMD Odyssey Windows Mixed Reality Headset with Motion Controllers Consultado el 10 de noviembre de 2017. Disponible en https://www.microsoft.com/en-us/store/d/samsung-hmd-odyssey-windows-mixed-reality-headsetwith-motion-controllers/8w91r774pdkk?activetab=pivot:overviewtab

- $\quad$ Sanz Rodriguez, Juan Carlos. El libro de la Imagen. Alianza Editorial, S.A., Madrid, 1996

- Scolari, Carlos Alberto. (2013). Narrativas Transmedia. Cuando todos los medios cuentan. Ed. DEUSTO. Centro Libros PAPF, S.L.U.

- Sony. Playstation-VR. Consultado el 5 de noviembre de 2017. Disponible en https://www.playstation.com/es-es/ explore/playstation-vr/

- SteamVR. . Consultado el 10 de noviembre de 2017.Véase http://store.steampowered.com/steamvr

- Thompson, J. B. (1998), Los media y la modernidad. Una teoría de los medios de comunicación, Barcelona, Paidós

- $\quad$ Virilio, Paul (2005). El cibermundo la política de lo peor. Editorial CATEDRA. ISBN: 9788437615745

- Wunderman. Marketing global. Un mano a mano con rance crain, editor in chief de ad age. Disponible en http:// www.adlatina.com/marketing/los-90-a\%C3\%B1os-la-leyenda-del-marketing-directo-lester-wunderman-habla-de-lapublicidad-del. Publicado el 28.07.2010. Consultado el 10 de noviembre de 2017.

- $\quad$ Xatakandroid. Todos los smartphones Android compatibles con Daydream VR y Project Tango. Consultado el 5 de noviembre de 2017. Disponible en https://www.xatakandroid.com/moviles-android/todos-los-smartphones-androidcompatibles-con-daydream-vr-y-project-tango

1 Disponible en http://www.samsung.com/global/galaxy/gear-vr/

2 Véase en https://www.vive.com/us/

3 Disponible en https://www.oculus.com/go/

4 Véase en https://www.oculus.com/rift/

5 Véase en http://b2b.ifa-berlin.com/en/IFA/IFASpecialAreas/IFANEXT/InnovationEngine/

6 Disponible en https://www.microsoft.com/en-us/hololens

7 Véase en https://www.youtube.com/watch?v=CjX2HI3pAgo

8 Véase en http://www.giztab.com/las-gafas-de-realidad-virtual-de-google-se-renuevan-con-android-vr/

9 Véase en https://www.xatakandroid.com/moviles-android/todos-los-smartphones-android-compatibles-con-daydreamvr-y-project-tango

10 Véase en http://www.nvidia.es/object/vr-ready-program-es.html

11 Véase en https://www.asus.com/microsite/beyond-vr-ready/ y https://www.youtube.com/watch?v=ZwxloWy2wTo

12 Véase en http://www.amd.com/es/technologies/vr-ready

13 Véase en https://www.playstation.com/es-es/explore/playstation-vr/

14 Véase http://store.steampowered.com/steamvr 
15 Consultable en https://www.microsoft.com/en-us/store/d/samsung-hmd-odyssey-windows-mixed-reality-headsetwith-motion-controllers/8w91r774pdkk?activetab=pivot:overviewtab

16 Disponible en https://www.facebook.com/spaces

17 Véase https://www.youtube.com/watch?time_continue=3\&v=OOuWJnR7iW0 y en http://www.adsoftheworld.com/campaign/caser-seguros-saatchi-saatchi-11-2017

18 Véase en "Marketing global. Un mano a mano con rance crain, editor in chief de ad age" http://www.adlatina.com/ marketing/los-90-a\%C3\%B1os-la-leyenda-del-marketing-directo-lester-wunderman-habla-de-la-publicidad-del. Publicado el 28.07.2010. 